\title{
Hybrid Visualization for White Matter Tracts using Triangle Strips and Point Sprites
}

\author{
Dorit Merhof, Markus Sonntag, Frank Enders, \\ Christopher Nimsky, Peter Hastreiter, Member, IEEE, and Günther Greiner
}

\begin{abstract}
Diffusion tensor imaging is of high value in neurosurgery, providing information about the location of white matter tracts in the human brain. For their reconstruction, streamline techniques commonly referred to as fiber tracking model the underlying fiber structures and have therefore gained interest. To meet the requirements of surgical planning and to overcome the visual limitations of line representations, a new real-time visualization approach of high visual quality is introduced. For this purpose, textured triangle strips and point sprites are combined in a hybrid strategy employing GPU programming. The triangle strips follow the fiber streamlines and are textured to obtain a tube-like appearance. A vertex program is used to orient the triangle strips towards the camera. In order to avoid triangle flipping in case of fiber segments where the viewing and segment direction are parallel, a correct visual representation is achieved in these areas by chains of point sprites. As a result, a high quality visualization similar to tubes is provided allowing for interactive multimodal inspection. Overall, the presented approach is faster than existing techniques of similar visualization quality and at the same time allows for real-time rendering of dense bundles encompassing a high number of fibers, which is of high importance for diagnosis and surgical planning.
\end{abstract}

Index Terms-Diffusion tensor data, fiber tracking, streamline visualization.

\section{INTRODUCTION}

Diffusion tensor imaging (DTI) is a magnetic resonance (MR) acquisition technique which has gained importance in recent years. It enables recovery of the organization and structure of white matter within the human brain in vivo which is of great interest for neuroscience. For this purpose, the diffusion property of water molecules is captured which is anisotropic in tissue with a high degree of directional organization such as white matter. The visualization of the resulting tensor data is non-trivial, leading to a wide variety of approaches. In principle, there are three major strategies for DTI visualization:

Glyph-based techniques aim at visualizing the whole tensor information per voxel. For this purpose, glyph representations such as ellipsoids [14] or superquadric tensor glyphs [19] are employed to convey the tensor information. Even though the tensor information is comprehensively represented using glyphs, the visualization results are difficult to interpret which makes them impractical for medical diagnosis and planning.

Alternative approaches use a diffusion metric to reduce the tensor information to a scalar value that provides a meaningful characteristic of the local diffusion. Several metrics have been presented such as fractional anisotropy (FA) [5] or the barycentric space coordinates $c_{l}$, $c_{p}, c_{s}$ [35] which characterize the shape of a diffusion tensor ( $l$ : linear, $p$ : planar, $s$ : spherical). With respect to medical application, 2D slice images of scalar metrics allow inspection of the local relation of

- Dorit Merhof is with Computer Graphics Group, Univ. Erlangen, and Dept. of Neurosurgery, Univ. Erlangen, E-mail:merhof@cs.fau.de.

- Markus W. Sonntag is with Computer Graphics Group, Univ. Erlangen, E-mail:sonntag@cs.fau.de.

- Frank Enders is with Computer Graphics Group, Univ. Erlangen, and Dept. of Neurosurgery, Univ. Erlangen, E-mail: enders@cs.fau.de.

- Christopher Nimsky is with Dept. of Neurosurgery, Univ. Erlangen, E-mail: christopher.nimsky@nch.imed.uni-erlangen.de.

- Peter Hastreiter is with Computer Graphics Group, Univ. Erlangen, and Dept. of Neurosurgery, Univ. Erlangen, E-mail: peter.hastreiter@neurozentrum.imed.uni-erlangen.de.

- Günther Greiner is with Computer Graphics Group, Univ. Erlangen, E-mail: greiner@cs.fau.de.

Manuscript received 31 March 2006; accepted 1 August 2006; posted online 6 November 2006.

For information on obtaining reprints of this article, please send e-mail to: tvcg@computer.org. white matter structures and neighboring anatomy and lesions. However, apart from the location of white matter tracts, their directional information is difficult to convey even if color coding according to the direction of main diffusion is applied.

Another class of algorithms known as fiber tracking [4, 28] relies on the major eigenvector of each tensor which indicates the direction of main diffusion. Most fiber tracking approaches utilize streamline techniques known from flow visualization to propagate fibers in the field of major eigenvectors. In this way, a much better understanding of the spatial relation between fibers and lesions is obtained. Recent research efforts in this field have turned fiber tracking into a powerful tool for the reconstruction of white matter tracts. For this reason, pioneering clinics have discovered fiber tracking as a valuable supplement in neurosurgery which contributes to make diagnosis and preoperative planning safer [29, 18, 7].

However, apart from exact tracking techniques, an efficient and meaningful visualization of the derived tracking results is of major interest for DTI analysis. Since the amount of streamlines may be immense in case of tracking the whole brain, and at the same time interactive visualization is necessary for medical investigation, appropriate techniques are required. Up to now, rendering of fiber tracking results is commonly accomplished using lines or tubes. However, simple line rendering implies several disadvantages. In case of curved fibers, gaps are visible between neighboring segments if the lines are wider than one pixel. Additionally, the depth perception is lost due to a constant width independent of the distance to the camera. These limitations are not encountered using tubes which provide a significantly improved visualization. However, the complex geometry of tubes makes rendering much more time consuming and therefore restricts interactivity. Alternatively, an approach based on triangle strips has been presented [25] which provides interactive rendering but suffers from artifacts when viewing direction and segment direction are parallel.

To overcome these limitations, we propose a hybrid visualization technique using a combination of textured triangle strips and point sprites which are textured depending on the viewing direction. As a result, a visualization very similar to tubes is obtained and the number of primitives is significantly reduced enabling interactive rendering. The presented strategy - interactive rendering of high quality images is of major interest for medical application in neurosurgery.

The paper is organized as follows: In Section 2, a survey of related work about fiber tracking and visualization of streamlines is given. 

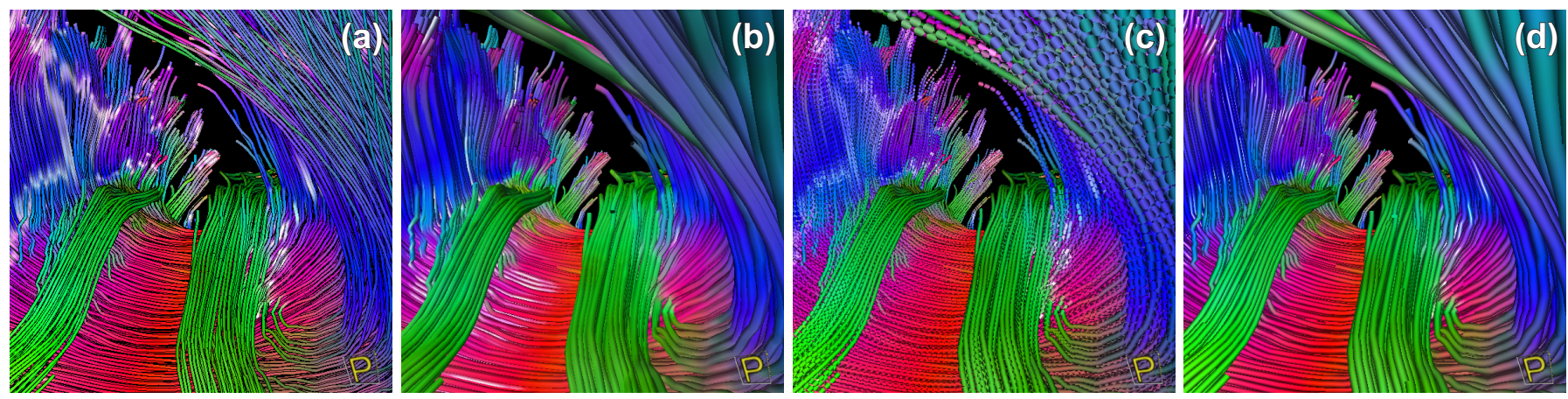

Fig. 1. Fiber tracking of the human brain showing a view across the corpus callosum, visualized with different rendering approaches. In comparison to rendering based on illuminated lines with halos $(a)$, geometry tubes $(b)$ or point sprites $(c)$, the presented hybrid approach based on triangle strips and point sprites $(d)$ provides a considerably improved visualization for more reliable medical diagnosis.

Section 3 gives an introduction to DTI data acquisition and preprocessing as well as fiber tracking. Section 4 is divided into three parts presenting the construction of triangle strips, point sprites and the hybrid rendering approach. An evaluation comparing computing times as well as a discussion is given in Section 5.

\section{Related WORK}

Fiber tracking approaches identify the location and orientation of fibrous tissue such as white matter tracts within the human brain. They have become a widely accepted technique for DTI visualization. This can also be attributed to the extensive research in this area making fiber tracking a powerful tool for white matter reconstruction. Research in this field aims at technical improvement of tracking algorithms, user-defined and automatic selection techniques, and visualization approaches.

With regard to fiber tracking, approaches based on streamline propagation $[4,28]$ are commonly employed. As an alternative, tracking techniques such as tensor deflection [21], wavefront evolution [16] or probabilistic tracking [6] have been presented. The objective of these more recent approaches is to track pathways also in subcortical or even cortical regions resulting in a large number of fiber streamlines. Since DTI data suffers from imaging noise, regularization of the tensor data either during tracking with moving least-squares filters [39] or based on the tensor field $[8,22]$, has been subject of current research. The accurate treatment of crossing and branching fibers is another important topic of research [32, 37, 34]. Recently, fiber tracking has been applied to higher order tensor data [15] providing a more comprehensive diffusion profile for each voxel. As a result, all tracking approaches provide pathways approximating the course and location of real nerve structures within the human brain.

Efficient tract selection approaches are an important tool to separate different tract systems which can be attributed to specific function. In medical application, regions of interest (ROIs) defined by a medical expert are typically used [29]. A similar technique based on dynamic queries $[2,9]$ enables selecting specific tract systems interactively from a tracking of the whole brain, using box-shaped ROIs. In addition to that, automatic techniques have emerged aiming at more convenient classification $[10,26]$. As a result, selection approaches provide mechanisms for fast and efficient extraction of separate tract systems such as the pyramidal tract, the optical tract or the corpus callosum contributing to fast and standardized tract retrieval in medical application.

With respect to visualization, the trajectories obtained by fiber tracking may be rendered using line based representations such as illuminated streamlines $[40,23]$. In this way, real-time rendering of a large number of trajectories is possible which is important for practical application since fiber trackings may result in several thousands of streamlines. As a drawback, depth information is not provided since lines are rendered with constant width. Furthermore, gaps be- tween adjacent segments are encountered if the width of the lines is larger than one pixel. To improve the visual perception and in order to provide a better overall impression, lines were extended to streamtubes [38]. Alternative approaches utilize hyperstreamlines [12, 36] to convey further tensor information through the shape of the tube in order to increase the information content. As a drawback, the resulting rendering times are considerably slower than in case of lines since complex geometry is necessary to model tubes or hyperstreamlines. In recent work, a more efficient technique for a tube-like visualization has been presented [33], combining a GPU-based approach using quad strips with a CPU-based tessellation in regions where artifacts occur. A speed-up of about 2.4 was achieved in comparison to approaches solely based on tessellated tubes. However, to meet the performance requirements encountered when rendering dense fiber tracts, this approach is still not fast enough due to a time-consuming CPU stage. An alternative technique for the visualization of DTI fiber tracts employs triangle strips [25] enabling interactive rendering. However, artifacts due to flipping triangles occur in regions where the fiber direction is nearly parallel to the viewing direction. Another technique based on cylindrical billboards [31] provides comparable results as long as thin streamlines are rendered.

Since interactivity is of crucial importance for the inspection of medical image data in practice, we present a new approach for the visualization of DTI fiber tracking which meets the clinical requirements. For this purpose, a pure GPU-based rendering strategy is introduced combining real-time performance and high visual quality. This is accomplished by textured triangle strips which are supplemented by point sprites to overcome artifacts. As a major achievement, realtime rendering is maintained and quality similar to tube representations is obtained. With respect to clinical application, the strategy has been applied in the context of multimodal visualization combining functional information showing white matter tracts and surrounding anatomy based on direct volume rendering of conventional MR data.

\section{DATA ACQUISITION AND PREPROCESSING}

From diffusion tensor image acquisition to fiber tracking, a number of processing steps are necessary. In this section, the principle of DTI is outlined as well as the subsequent data processing. Finally, an introduction to fiber tracking is provided.

\subsection{Image data}

DTI is a magnetic resonance acquisition technique which has gained considerable attention in recent years. It measures the diffusion of water molecules, also known as Brownian motion [13]. Depending on the shape of the cells and properties like the permeability of the cell membranes, diffusion varies significantly. As a result, the diffusion characteristics measured for each voxel reveal information about the underlying tissue structure. In particular, water molecules within white 
matter feature strong anisotropic diffusion since the long, cylindric nerve cells restrict diffusion to their developing direction.

Based on a reference dataset measured without gradient and six datasets acquired for different gradient directions, a symmetric second order tensor is computed for each voxel [3]:

$$
D=\left(\begin{array}{ccc}
D_{x x} & D_{x y} & D_{x z} \\
D_{x y} & D_{y y} & D_{y z} \\
D_{x z} & D_{y z} & D_{z z}
\end{array}\right)
$$

The tensor characterizes the properties of local diffusion whereas the direction of the major eigenvector indicates the direction of main diffusion. The eigenvalues $\lambda_{i}, i=1,2,3$ give information about the anisotropy of a tensor which is an indicator for white matter structures. In case of eigenvalues with similar size $\lambda_{1} \approx \lambda_{2} \approx \lambda_{3}$, isotropic diffusion is encountered which occurs in tissue where the cells have a round shape or in fluid filled cavities like the ventricles. If one eigenvalue dominates over the others, i.e. $\lambda_{1} \gg \lambda_{2} \approx \lambda_{3}$, white matter structures are encountered showing a strong anisotropic diffusion characteristic. In case of crossing or branching fibers, two eigenvalues of comparable size are much greater than the third one $\lambda_{1} \approx \lambda_{2} \gg \lambda_{3}$.

The datasets employed in this work were acquired in patients with brain tumors undergoing surgery. All the datasets used in this work were measured using a Siemens MR Magnetom Sonata Maestro Class 1.5 Tesla scanner. The specifications of the gradient system were a field strength of up to $40 \mathrm{mT} / \mathrm{m}$ (effective $69 \mathrm{mT} / \mathrm{m}$ ) and a slew rate of up to $200 \mathrm{~T} / \mathrm{m} / \mathrm{s}$ (effective $346 \mathrm{~T} / \mathrm{m} / \mathrm{s}$ ) for clinical application. We chose a field of view of $240 \mathrm{~mm}$ resulting in a voxel size of $1.875 \times 1.875 \times 1.9 \mathrm{~mm}^{3}$. For each of the six gradient directions $( \pm 1,1,0),( \pm 1,0,1),(0,1, \pm 1)$ and the reference image sixty slices with no intersection gap and an acquisition matrix of $128 \times 128$ were measured. Additionally, a $\mathrm{MRI}_{T 1}$ sequence $(256 \times 256 \times 160$ voxels $)$ was acquired in each patient which was used for surgical planning.

\subsection{Fiber tracking}

In general, tracking algorithms based on streamline propagation have several steps in common such as seed point selection, fiber propagation and termination strategies [28]. Starting from predefined seed points or ROIs, streamline integration is used to propagate the fiber until a termination criterion is reached. For this purpose, a threshold based on fractional anisotropy (FA) $[4,27]$ is commonly applied to stop fiber propagation if the direction of dominant diffusion is not well defined. In some approaches, a further threshold based on curvature [4] is employed which follows the assumption that fibers are somewhat stiff. This prevents the tracking algorithm from following unlikely paths.

For the definition of seed points different strategies exist: Seeds may be automatically located in regions with high anisotropy and used for fiber tracking within the whole brain. If ROIs are additionally specified, only those fibers are displayed which cross these regions. As an advantage of this strategy, fibers within the whole brain are precomputed and can then be subject to different dynamic queries [2]. Alternatively, seed points are directly positioned within the ROI which consumes less computation time. However, if different tract systems of the same subject have to be investigated, the first approach is more efficient. Furthermore, if the ROI is located in subcortical or cortical areas where the direction of diffusion is typically less pronounced, the tracking result will be more reliable if tracking is started in regions of high anisotropic diffusion.

In this work, fiber propagation is accomplished using streamline approaches known from computational fluid dynamics. For this purpose, trilinear tensor interpolation, computation of the eigensystem and numerical integration are necessary in each propagation step. In order to derive a tensor at arbitrary positions within the dataset, componentwise trilinear interpolation of the tensor entries is used. Previous research has shown that component-wise trilinear interpolation provides better results than interpolation of the principal eigenvectors [20]. Another processing step comprises the computation of the eigensystem of the interpolated tensor to obtain the major eigenvector which correlates with the dominant direction of diffusion. In order to determine the direction of streamline propagation, higher order integration schemes requiring repeated tensor interpolation and eigensystem computation are applied. We used the fourth-order Runge-Kutta method providing sufficient accuracy. An outline of streamline propagation is given in Figure 2.

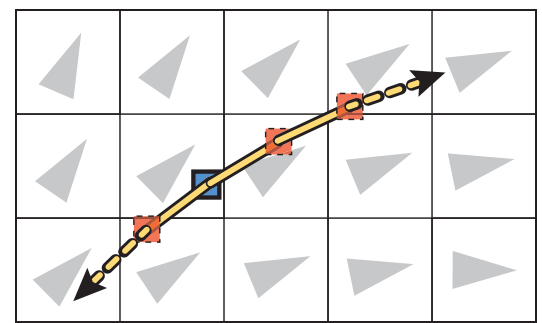

Fig. 2. Schematic outline of fiber tracking based on streamline propagation. According to the underlying diffusion direction, each streamline is pursued in forward and backward direction until a termination criterion is reached.

\section{HYBRID VISUALIZATION USING TRIANGLE STRIPS AND POINT SPRITES}

Current visualization techniques for streamlines either provide high visual quality, but are very time consuming when fibers of the whole brain are rendered, or reduced quality has to be accepted for interactive visualization. In this paper, a hybrid approach combining triangle strips and point sprites is presented which meets the clinical requirements for visualizing a large number of fiber tracts. That is, real-time rendering is achieved and at the same time the visualization quality is comparable to geometry-based tubes.

\subsection{Visualization using triangle strips}

The visualization of streamlines using geometry tubes provides high quality. However, the approach becomes slow if the number of fibers increases due to the corresponding large number of triangles. Alternative techniques [33] achieving more efficiency are still not fast enough to render fiber trackings of the brain encountered in clinical application

The crucial advantage of triangle strips over tube-based representations is the fact that the geometry is considerably reduced. Instead of modeling a tracking segment with triangles forming a tube, only two triangles per segment are required in our approach. The triangles are rotated towards the camera by a vertex program and are textured in order to obtain a tube-like appearance. Furthermore, the applied triangle strips provide a depth dependent width which supports the visual perception.

The reconstruction of the triangle strip is outlined in Figure 3. Starting from each sampling point $P_{i}$ of the streamline, two new vertices are

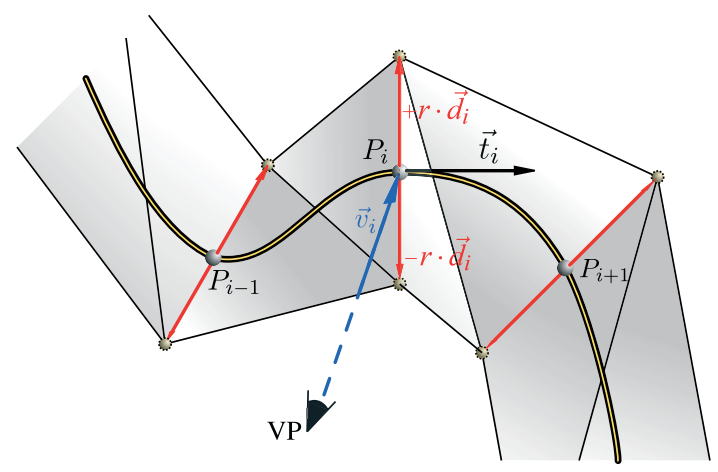

Fig. 3. Construction of a triangle strip. Based on sampling nodes of streamline (yellow), nodes for modeling the triangle strip are generated. 
generated which are used as boundary vertices of the triangle strip. For this purpose, the cross product $\vec{d}_{i}=\vec{v}_{i} \times \vec{t}_{i}$ between viewing direction $\vec{v}_{i}$ and streamline tangent $\vec{t}_{i}$ is computed providing the direction $\vec{d}_{i}$ which is used to place the new nodes. The width of the tube is defined by the user by choosing the radius $r . \vec{d}_{i}$ is normalized and the new nodes $P_{i, \text { north }}, P_{i, \text { south }}$ necessary to construct the triangle strip are generated:

$$
P_{i, \text { north } / \text { south }}=P_{i} \pm r \cdot \vec{d}_{i} \text { with } \vec{d}_{i}=\frac{\vec{v}_{i} \times \vec{t}_{i}}{\left\|\vec{v}_{i} \times \vec{t}_{i}\right\|}
$$

The construction of the triangle strip is efficiently computed using graphics hardware. For this purpose, the triangle strip contains each node $P_{i}$ twice since the shifted node locations are computed for each frame within the vertex program. For each copy of $P_{i}$, a 2D texture coordinate is stored where the first coordinate corresponds to a 1D texture used for simulating tube-like illumination. The second $2 \mathrm{D}$ texture coordinate contains a flag which is 1 if the node is shifted to north resulting in $P_{i, n o r t h}$, and -1 if the node is shifted to south, respectively. In Figure 4, the texture coordinates are given in brackets for each node of the triangle strip.

An essential component for a tube-like appearance of a triangle strip is adequate texturing which simulates illumination of a tube. For this purpose, a 1D texture with a luminance and an alpha channel is employed. The luminance channel stores the diffuse illumination whereas the alpha channel contains the specular term. In order to simulate illumination of a tube, the textures have to get darker when approaching the border of the triangle strip. In case of diffuse illumination, a sine function for angles between 0 and 180 degrees provides the appropriate decay. For specular highlights, the bright spot has to be focused. Good results were obtained by raising the values for diffuse illumination to a power of 16 . Figure 4 shows both channels of the texture on the left and a textured triangle strip segment on the right. In order to obtain a smooth illumination effect, the normals in viewing direction at two adjacent sampling points $P_{i}$ and $P_{i+1}$ are interpolated.
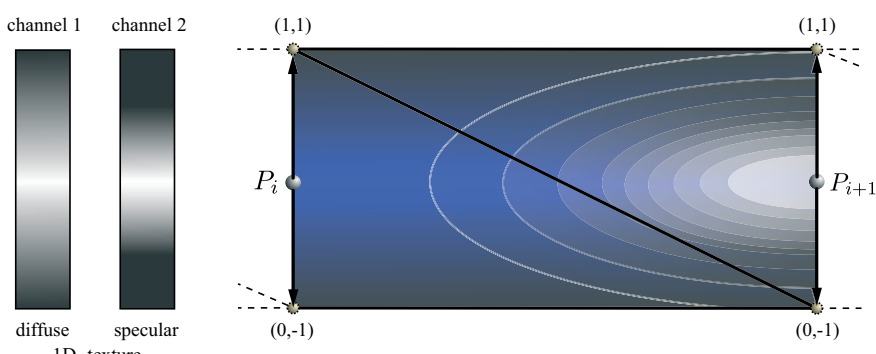

Fig. 4. A 1D texture is used to simulate tube-like illumination. The luminance channel is used for diffuse illumination, the alpha channel stores the specular term.

The underlying color of the textures is based on the standard color scheme commonly used in DTI $[11,17,30]$. The spatial direction of each fiber segment is color encoded assigning a RGB color value to the xyz component of the normalized tangent. As a result, the orientation of fibers is additionally highlighted by color, and fibers of similar direction are visually bundled.

For efficient rendering using OpenGL, all tracked streamlines are drawn by invoking GL_TRIANGLE_STRIP only once. In order to obtain separated streamlines at the end, degenerate triangles are employed which connect two streamlines by positioning all triangle points on a line. Thus OpenGL excludes these triangles from rendering. For optimum rendering performance, the nodes and their attributes are stored interleaved using a vertex buffer object (VBO).

\subsubsection{Flipping artifacts}

The presented approach based on triangle strips provides good results as long as the direction of a line segment does not come close to the viewing direction. Otherwise, flipping artifacts may occur when viewing and segment direction are nearly parallel. Artifacts arising from flipping triangles are shown in Figure 5.

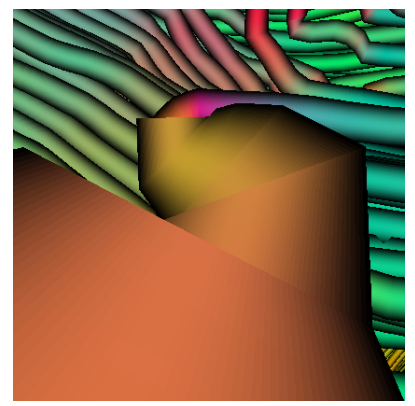

Fig. 5. Artifacts originating from flipping triangles are observed if the viewing direction and segment direction are nearly parallel.

\subsection{Visualization using point sprites}

Point sprites are particularly suitable for the visualization of line segments which are nearly parallel to the viewing direction. In this case, they provide a natural, tube-like visualization since they resemble the cross section of a tube. A point sprite corresponds to a textured square which is always oriented towards the camera and which is efficiently visualized using the OpenGL extension for point sprites. Similar to textured triangle strips, a $64 \times 642 \mathrm{D}$-RGB-texture is used to draw and illuminate these sprites (see Figure 6). The R-channel is used as alpha channel to obtain a spherical shape of the point sprite. The $\mathrm{G}$ and B-channel are used to simulate diffuse and specular illumination.

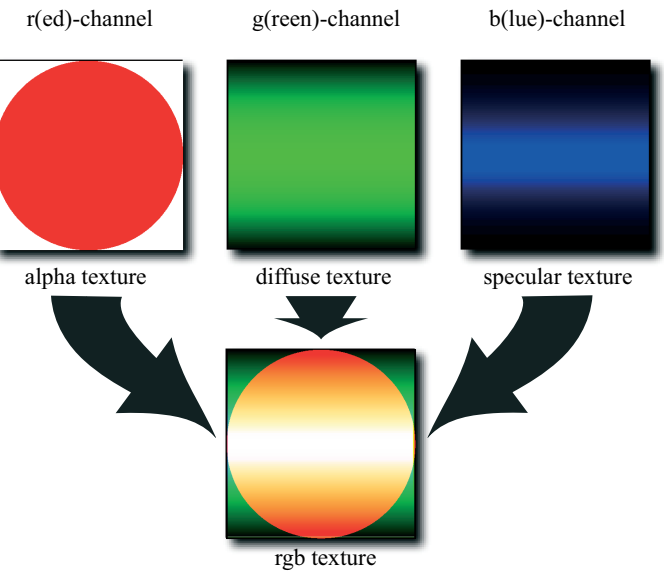

Fig. 6. The channels of a RGB-texture are used to draw and illuminate a point sprite. The R-channel is used as alpha channel, the G- and $\mathrm{B}$-channel are used to simulate diffuse and specular illumination.

In order to obtain a tube-like appearance, the point sprites have to be rotated according to the direction of the streamline segments. This is achieved by rotating the texture coordinates of the point sprite. The rotation of a point sprite positioned at location $P_{1}$ depends on the direction of the tangent at this streamline point. In order to obtain the parameters needed for the rotation, the tangent $\vec{t}$ at point $P_{1}$ has to be projected to the image plane resulting in $\vec{t}_{p}$ (compare Figure 7). This is achieved by first multiplying $P_{1}$ and $P_{2}=P_{1}+\vec{t}$ with the model view projection matrix $\mathbf{M}_{4 \times 4}$ :

$$
\begin{aligned}
& P_{1 p} . x y z w=\mathbf{M}_{4 \times 4} \cdot P_{1} \cdot x y z w \\
& P_{2 p} . x y z w=\mathbf{M}_{4 \times 4} \cdot P_{2} \cdot x y z w
\end{aligned}
$$


The projection $\vec{t}_{p}$ of the tangent vector $\vec{t}$ into the image plane is then obtained by dehomogenization and conversion to screen coordinates:

$$
\vec{t}_{p} \cdot x y=\left(\begin{array}{c}
P_{2 p} \cdot x / P_{2 p} \cdot w-P_{1 p} \cdot x / P_{1 p} \cdot w \\
-P_{2 p} \cdot y / P_{2 p} \cdot w+P_{1 p} \cdot y / P_{1 p} \cdot w
\end{array}\right) .
$$

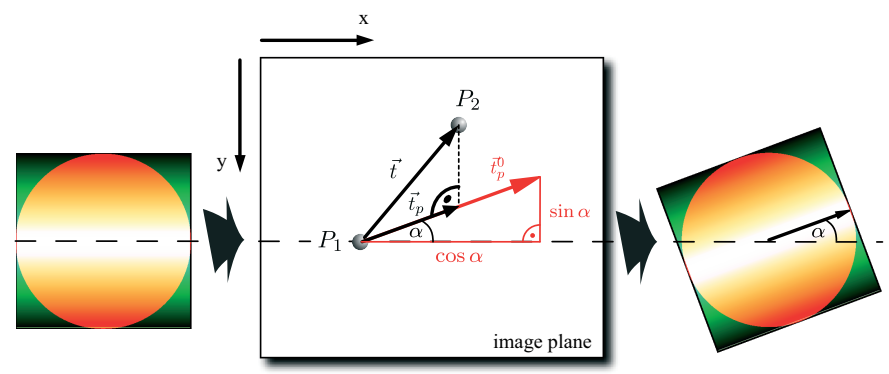

Fig. 7. Rotation of the 2D texture coordinates depending on the angle $\alpha$. The parameters for specifying the rotation are obtained from the tangent vector $\vec{t}$ which is projected into the image plane and normalized resulting in $\vec{t}_{p}^{0}$.

Normalization of $\vec{t}_{p}$ results in a vector $\vec{t}_{p}^{0}=\left(\begin{array}{c}\cos \alpha \\ \sin \alpha\end{array}\right)$ where the angle $\alpha$ denotes the rotation of the segment with respect to the $\mathrm{x}$-axis (see Figure 8). The components of $\vec{t}_{p}^{0}$ which are computed by a vertex program are then used within a fragment program to compute the rotation of the texture coordinates. Since the texture coordinates T.xy are defined within $[0 ; 1]^{2}$ and the rotation is defined within $[-1 ; 1]^{2}$, each texture coordinate is first translated by $(-0.5 ;-0.5)$. Having applied the rotation, they are shifted back to their original domain:

$$
\begin{aligned}
& T_{\text {new }} \cdot x=\left(\vec{t}_{p}^{0} \cdot y \cdot(T \cdot y-0.5)+\vec{t}_{p}^{0} \cdot x \cdot(T \cdot x-0.5)\right)+0.5 \\
& T_{\text {new }} \cdot y=\left(\vec{t}_{p}^{0} \cdot y \cdot(T \cdot x-0.5)-\vec{t}_{p}^{0} \cdot x \cdot(T \cdot y-0.5)\right)+0.5
\end{aligned}
$$

The rotation is computed for each fragment resulting in correctly oriented point sprites. The approach provides a good visual representation for segments where the viewing direction is nearly parallel to the segment direction as illustrated in Figure 8.
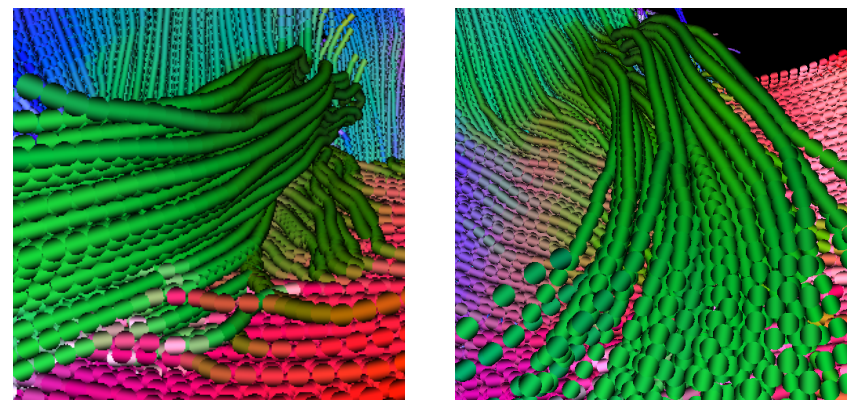

Fig. 8. Visualization of fiber tracts using point sprites with textures rotated according to the respective segment direction. Good visual results are obtained for segments nearly parallel to the viewing direction whereas the quality decreases for more perpendicular directions.

\subsection{Hybrid visualization using triangle strips and point sprites}

As long as a streamline is oriented perpendicular to the viewing direction, the profile of the tube is rectangular (Figure 9 (left)) which is appropriately visualized using triangle strips. However, if the viewing direction is nearly parallel to the direction of a segment, the profile of a tube is better represented using a circle (Figure 9 (right)) or a point sprite, respectively. For this reason, the presented hybrid approach combines the advantages of triangle strips and point sprites leading to a comprehensive and efficient visualization.
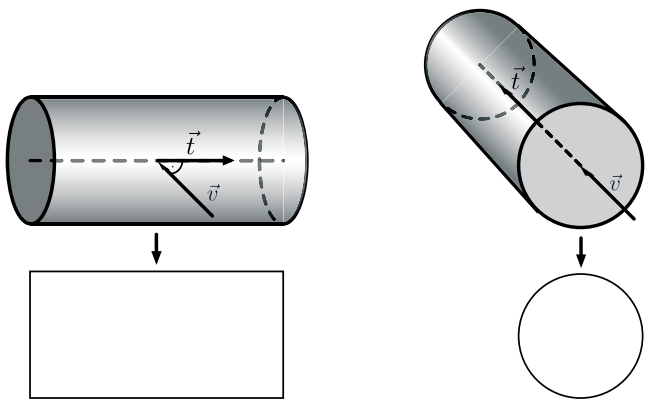

Fig. 9. Profile of a tube: Rectangular when viewing direction $\vec{v}$ perpendicular to fiber tangent $\vec{t}$ (left), spherical in case of viewing direction $\vec{v}$ parallel to tangent $\vec{t}$.

Within the hybrid approach, the size of the point sprite is computed to fit the width of the triangle strip. Accordingly, the point sprite is scaled to the required size within the vertex program using glPoint Size (). Depending on the scalar product $\langle\vec{t}, \vec{v}\rangle$ between tangent vector $\vec{t}$ and viewing direction $\vec{v}$, a decision is made whether the point sprite is displayed. More precisely, the point sprite is visualized if the scalar product is greater than 0.93 , otherwise the homogeneous coordinate of the point is set to zero and the point is clipped within the rendering pipeline. In order to obtain an overlap region of point sprites and triangle strips, the latter are displayed if the scalar product is less than 0.98 . Otherwise, drawing of triangle strips is suppressed by setting the A component of the RGBA color value to zero. The transparency value (A value) is checked for each fragment using glAlphaFunc preventing the visualization from flipping triangles. As a result, rendering based on triangle strips only leads to flipping artifacts whereas the hybrid approach incorporating point sprites provides a tube-like visualization without artifacts (see Figure 10).
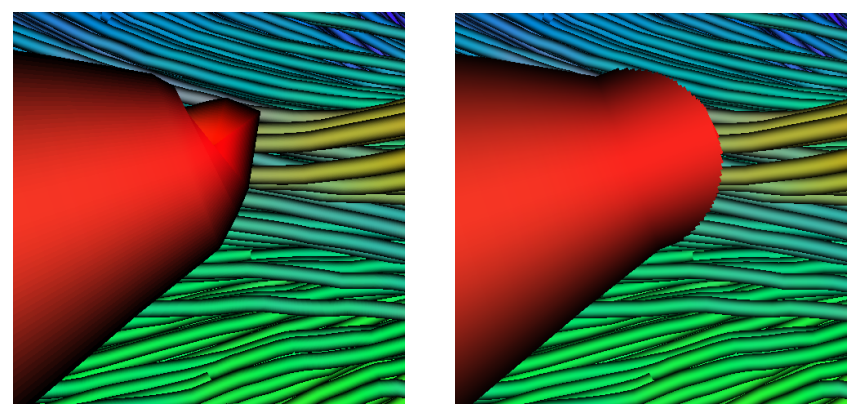

Fig. 10. Flipping triangles for a segment in viewing direction (left), these artifacts are overcome by the hybrid approach incorporating point sprites (right).

\subsubsection{Terminating textured quads}

The presented technique provides a natural appearance which is very similar to tubes. However, at the beginning and the end of each fiber, the representation lacks realism since a tube-like ending is missing and the flat geometry of the triangle strip becomes apparent. In order to overcome this drawback, a terminating textured quad is used at both ends of each fiber (compare Figure 11). 

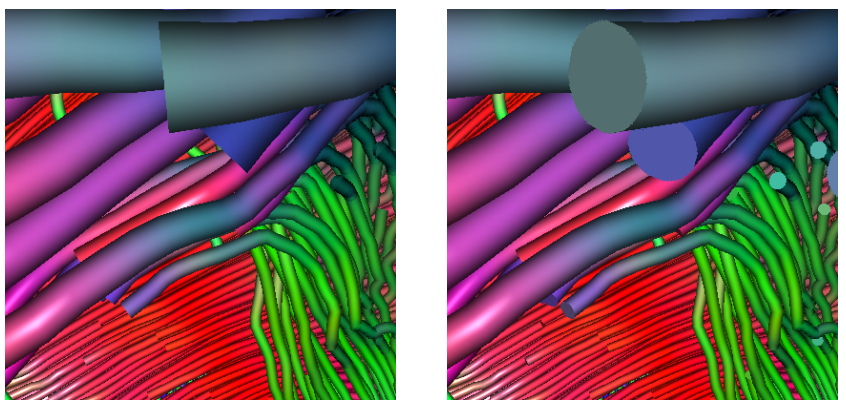

Fig. 11. More realistic representation: Hybrid approach without (left) and with terminating textured quads at both ends of each fiber (right).

\section{Results AND discussion}

The algorithms developed in this work were implemented using OpenGL and GLSL. For benchmarking, a PC equipped with a P4 3.0 GHz, 2 GB RAM and a NVidia Geforce 6800 Ultra graphics card with $256 \mathrm{MB}$ graphics memory was used. The viewport for all renderings was $600 \times 800$ pixels and the displayed set of lines, generated by fiber tracking within the whole brain, consisted of approximately 300000 segments originating from about 4500 fibers.

Table 1 gives a detailed overview of the performance of the presented methods. Lines and tubes are the geometrical approaches where a single tube segment consists of 16 triangles. They were compared to triangle strips, point sprites and the presented combination of these two techniques. All approaches were analyzed with respect to Vertex Buffer Objects (VBOs) provided by OpenGL for optimized geometry transfer. Illumination implemented in a fragment program was considered separately for each strategy. The performance measurements provided in Table 1 show that the presented hybrid approach achieves interactive frame rates regardless of the massive load of $300000 \mathrm{seg}$ ments encountered for a whole brain tracking.

\begin{tabular}{lr}
\hline Visualization Technique & VBOs \\
\hline Lines (no illumination) & $242.5 \mathrm{fps}$ \\
Lines (illuminated) & $153.3 \mathrm{fps}$ \\
Tubes (no illumination) & $7.5 \mathrm{fps}$ \\
Tubes (illuminated) & $7.5 \mathrm{fps}$ \\
Triangle Strips (no illumination) & $85.3 \mathrm{fps}$ \\
Triangle Strips (illuminated) & $65.4 \mathrm{fps}$ \\
Point Sprites (no illumination, no rotation) & $203.2 \mathrm{fps}$ \\
Point Sprites (illumination, rotation) & $89.5 \mathrm{fps}$ \\
Triangle Strips \& Point Sprites (no illumination) & $57.5 \mathrm{fps}$ \\
Triangle Strips \& Point Sprites (illumination) & $42.5 \mathrm{fps}$ \\
\hline
\end{tabular}

Table 1. Frame rates in fps (frames per second) depending on the visualization technique and illumination.

Using geometrically modeled tubes, the geometry load was about 4.8 million triangles per frame which led to 4.2 million vertices in case of triangle strip rendering. According to our observations, the sampling rate for tubes should not be less than 8 resulting in an octagon profile in order to obtain acceptable rendering quality. A further increase of the resolution leading to a higher number of triangles would significantly reduce the rendering performance since the vertex load becomes the limiting factor. Similarly, a higher number of fibers which is encountered in case of probabilistic tracking techniques or for dense fiber trackings, would decrease the rendering performance even more if geometry-based tube representations are used.

In comparison to geometric tubes, rendering times are about six (with illumination) to eight (no illumination) times faster using the suggested hybrid approach enabling interactive visualization at high quality. The high number of triangles necessary in case of conventional tube representations is reduced by a factor of eight, which leads to less geometric primitives and consequently to higher performance. As a result, the presented purely GPU-based approach overcomes the limitations of standard geometry-based approaches and provides further speed-up compared to a hybrid CPU-GPU-based approach [33]. At the same time the rendering quality is preserved.

Considering practical application, accelerated rendering of tubelike streamlines is an important prerequisite for a fusion with anatomical data based on direct volume rendering (DVR). Since DVR is very demanding in terms of rasterization power, it is essential to reduce the effort for additional rendering tasks (e.g. geometry) to a minimum. Due to the significantly reduced amount of data using the presented hybrid visualization technique, multimodal inspection of functional and anatomical information is possible in real-time. As an important result, functional data such as fiber tracts are explored within their anatomical context. Critical areas can be investigated in a convenient way (e.g. a tumor in the ultimate neighborhood to neuronal pathways) which contributes to optimized diagnosis and considerably improved therapy planning.

As can be seen in Figure 12, multimodal visualization combining function and surrounding anatomy were applied in a patient with a brain tumor. The overview figures show the global location of the tumor whereas the zoomed views provide details of white matter structures surrounding the tumor. Investigating such a multimodal representation, the surgeon is able to verify whether important tract systems are located in the vicinity of the tumor. Based on this information, an appropriate surgery plan can be developed. Since the neuronal structures have to remain intact to avoid postoperative neurological deficits, their real-time visualization together with anatomical data is an important assistance for safer surgery. Overall, the presented technique provides visualization of high quality and at the same time enables better inspection due to high frame rates. It is a valuable supplement for medical application in neurosurgery.

\section{Conclusion}

In this work, a novel visualization technique for DTI fiber tracts has been presented. Its development was encouraged by the demands of neurosurgery where interactive multimodal visualization of high quality is required. The presented solution introduces a hybrid, purely GPU-based rendering approach incorporating textured triangle strips and point sprites. By combining the advantages of both techniques, tube-like representations are obtained at real-time frame rates. As a major achievement, the approach is directly applicable in clinical practice, providing a comprehensive and meaningful visualization for fiber tracts obtained from DTI data. Overall, the visualization strategy effectively contributes to improve medical diagnosis and therapy planning in neurosurgery.

\section{FUTURE WORK}

The presented hybrid rendering approach is a valuable alternative to line based or sparse tube based representations currently applied for DTI fiber tract visualization. The visual effect of depth perception could be further intensified with shadows. This could be accomplished using a fragment program for shadow maps which computes depth values of the fiber segments with respect to a light source. Though this would lower the frame rates, it could be an option for trackings of lower density or single tract systems.

\section{ACKNOWLEDGEMENTS}

This work was supported by the Deutsche Forschungsgemeinschaft in the context of SFB 603, Project C9.

\section{REFERENCES}

[1] OpenGL(R) Shading Language. Addison Wesley Longman, 2004.

[2] D. Akers, A. Sherbondy, R. Mackenzie, R. Dougherty, and B. Wandell. Exploration of the Brain's White Matter Pathways with Dynamic Queries. In IEEE Visualization, pages 377-384, 2004.

[3] P. Basser, J. Mattiello, and D. LeBihan. Mr diffusion tensor spectroscopy and imaging. Biophys J, 66(1):259-267, 1994. 

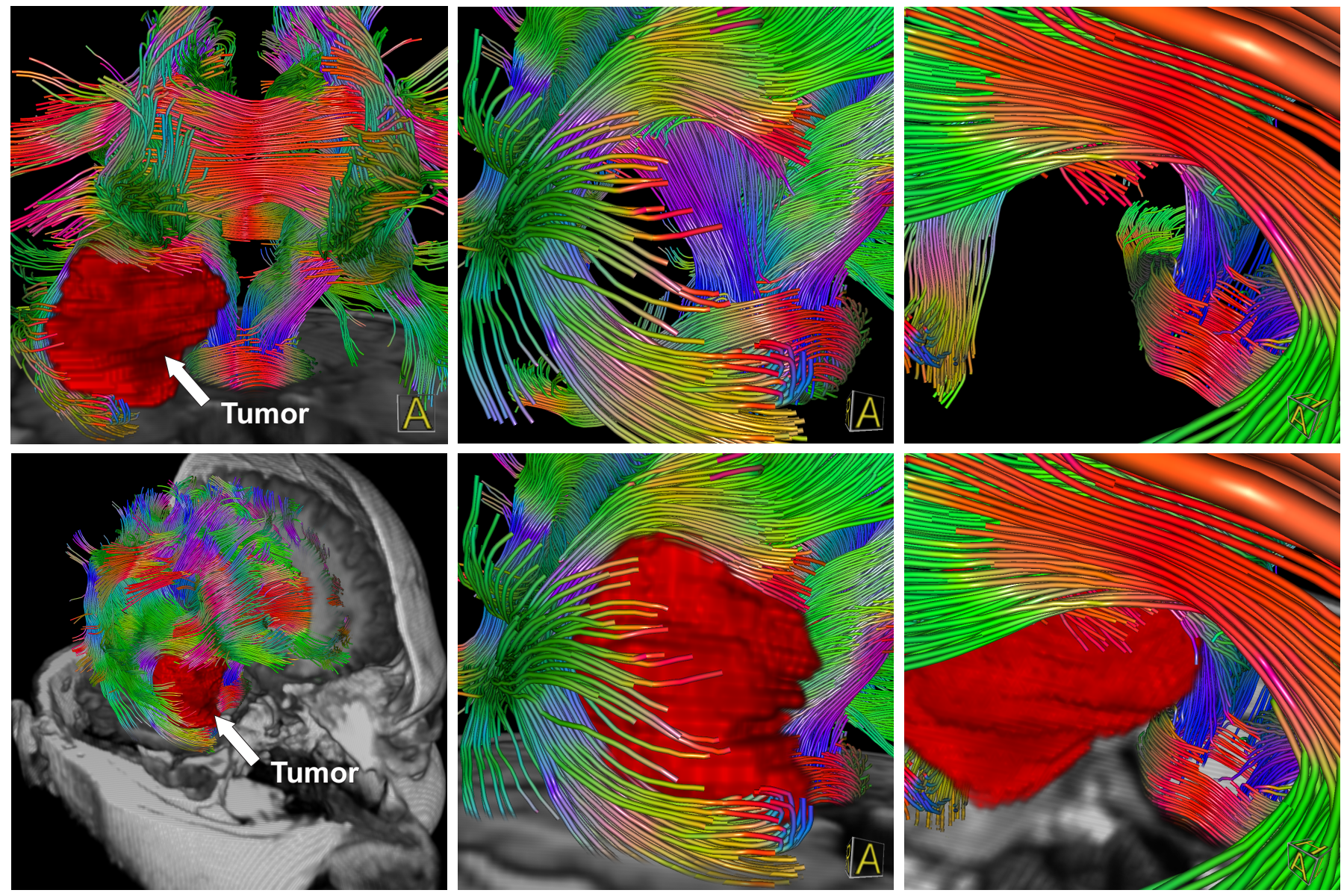

Fig. 12. Fiber tracking in a patient with a brain tumor (red) rendered with the presented hybrid approach. Additionally, direct volume rendering of anatomical data provides background information about the location of the neuronal pathways with respect to the tumor and the surrounding anatomy. Close-up views of fibers (middle, right) and comparison of rendering with and without tumor (compare upper and lower row) provide important information for surgical planning.

[4] P. Basser, S. Pajevic, C. Pierpaoli, J. Duda, and A. Aldroubi. In vivo fiber tractography using DT-MRI data. Magnet Reson Med, 44:625-632, 2000.

[5] P. Basser and C. Pierpaoli. Microstructural and physiological features of tissues elucidated by quantitative-diffusion-tensor MRI. J Magn Reson, Series B, 111(3):209-219, 1996.

[6] T. Behrens, H. Johansen-Berg, M. Woolrich, S. Smith, C. WheelerKingshott, P. Boulby, G. Barker, E. Sillery, K. Sheehan, O. Ciccarelli, A. Thompson, J. Brady, and P. Matthews. Non-invasive mapping of connections between human thalamus and cortex using diffusion imaging. Nat Neurosci, 6(7):750-757, 2003

[7] J. Berman, M. Berger, P. Mukherjee, and R. Henry. Diffusion-tensor imaging-guided tracking of fibers of the pyramidal tract combined with intraoperative cortical stimulation mapping in patients with gliomas. Neurosurgery, 101(1):66-72, 2004.

[8] M. Björnemo, A. Brun, R. Kikinis, and C.-F. Westin. Regularized Stochastic White Matter Tractography Using Diffusion Tensor MRI. In MICCAI, pages 435-442, 2002.

[9] J. Blaas, C. Botha, F. Vos, and F. Post. Fast and Reproducible Fibre Bundle Selection in DTI Visualization. In IEEE Visualization, pages 5964, 2005.

[10] A. Brun, H. Knutsson, H.-J. Park, M. Shenton, and C.-F. Westin. Clustering Fiber Tracts Using Normalized Cuts. In MICCAI, LNCS, pages 368-375. Springer, 2004.

[11] J. Coremans, R. Luypaert, F. Verhelle, T. Stadnik, and M. Osteaux. A Method for Myelin Fiber Orientation Mapping Using Diffusion-Weighted MR Images. Magn Reson Imaging, pages 443-454, 1994.

[12] T. Delmarcelle and L. Hesselink. Visualizing Second-Order Tensor Fields with Hyperstreamlines. IEEE Comput Graph, 13(4):25-33, 1993.
[13] A. Einstein. Über die von der molekularkinetischen Theorie der Wärme geforderte Bewegung von in ruhenden Fluessigkeiten suspendierten Teilchen. Annalen der Physik und Chemie, IV. Folge, 17:549-560, 1905.

[14] F. Enders, S. Iserhardt-Bauer, P. Hastreiter, C. Nimsky, and T. Ertl. Hardware-Accelerated Glyph Based Visualization of Major White Matter Tracts for Analysis of Brain Tumors. In Medical Imaging. SPIE, 2005.

[15] M. Hlawitschka and G. Scheuermann. HOT Lines: Tracking Lines in Higher Order Tensor Fields. In IEEE Visualization, pages 27-34, 2005.

[16] M. Jackowski, C. Kao, M. Qiu, R. Constable, and L. Staib. White matter tractography by anisotropic wavefront evolution and diffusion tensor imaging. Med Image Anal, 9:427-440, 2005.

[17] D. Jones, S. Williams, and M. Horsfield. Full Representation of WhiteMatter Fibre Direction in One Map via Diffusion Tensor Analysis. In Proc Int Soc Mag Res in Med, page 1743, 1997.

[18] K. Kamada, T. Todo, Y. Masutani, S. Aoki, K. Ino, T. Takano, T. Kirino, N. Kawahara, and A. Morita. Combined use of tractography-integrated functional neuronavigation and direct fiber stimulation. Neurosurgery, 102(4):664-627, 2005

[19] G. Kindlmann. Superquadric Tensor Glyphs. In EG/IEEE TCVG VisSym, pages 147-154, 2004.

[20] G. Kindlmann, D. Weinstein, and D. Hart. Strategies for Direct Volume Rendering of Diffusion Tensor Fields. IEEE T Vis Comput Gr, 6(2):124138,2000

[21] M. Lazar, D. Weinstein, J. Tsuruda, K. Hasan, K. Arfanakis, M. Meyerand, B. Badie, H. Rowley, V. Haughton, A. Field, and A. Alexander. White Matter Tractography Using Diffusion Tensor Deflection. Hum Brain Mapp, 18(4):306-321, 2003.

[22] C. A.-L. M. Martin-Fernandez, C.-F. Westin. 3D Bayesian Regularization 
of Diffusion Tensor MRI using Multivariate Gaussian Markov Random Fields. In MICCAI, LNCS, pages 351-359. Springer, 2004.

[23] O. Mallo, R. Peikert, C. Sigg, and F. Sadlo. Illuminated Lines Revisited. In IEEE Visualization, pages 19-25, 2005.

[24] O. Mattausch, T. Theuß1, H. Hauser, and E. Gröller. Strategies for interactive exploration of 3D flow using evenly-spaced illuminated streamlines. Spring Conference on Computer Graphics (SCCG), pages 213222, 2003.

[25] D. Merhof, M. Sonntag, F. Enders, C. Nimsky, P. Hastreiter, and G. Greiner. Streamline visualization of diffusion tensor data based on triangle strips. In Bildverarbeitung für die Medizin (BVM), Informatik Aktuell, pages 271-275. Springer, 2006.

[26] B. Moberts, A. Vilanova, and J. van Wijk. Evaluation of Fiber Clustering Methods for Diffusion Tensor Imaging. In IEEE Visualization, pages 6572, 2005 .

[27] S. Mori, B. Crain, V. Chacko, and P. van Zijl. Three Dimensional Tracking of Axonal Projections in the Brain by Magnetic Resonance Imaging. Ann Neurol, 45(2):265-269, 1999.

[28] S. Mori and P. van Zijl. Fiber tracking: principles and strategies - a technical review. NMR Biomed, 15:468-480, 2002.

[29] C. Nimsky, O. Ganslandt, P. Hastreiter, R. Wang, T. Benner, A. Sorensen, and R. Fahlbusch. Preoperative and intraoperative diffusion tensor imaging-based fiber tracking in glioma surgery. Neurosurgery, 56(1):130-138, 2005.

[30] S. Pajevic and C. Pierpaoli. Color schemes to represent the orientation of anisotropic tissues from diffusion tensor data: Application to white matter fiber tract mapping in the human brain. Magn Reson Imaging, 42(3):526-540, 1999.

[31] M. Schirski, T. Kuhlen, M. Hopp, P. Adomeit, S. Pischinger, and C. Bischof. Virtual Tubelets - efficiently visualizing large amounts of particle trajectories. Computers \& Graphics, 29(1):17-27, 2005.

[32] P. Staempfli, T. Jaermann, G. Crelier, S. Kollias, A. Valavanis, and P. Boesiger. Resolving fiber crossing using advanced fast marching tractography based on diffusion tensor imaging. Neuroimage, 30(1):110-120, 2006.

[33] C. Stoll, S. Gumhold, and H.-P. Seidel. Visualization with stylized line primitives. In IEEE Visualization, pages 695-702, 2005.

[34] A. Vilanova, G. Berenschot, and C. van Pul. DTI Visualization with Streamsurfaces and Evenly-Spaced Volume Seeding. In EG/IEEE TCVG VisSym, pages 173-182, 2004.

[35] C.-F. Westin, S. E. Maier, H. Mamata, A. Nabavi, F. A. Jolesz, and R. Kikinis. Processing and Visualization of Diffusion Tensor MRI. Med Image Anal, 6(2):93-108, 2002.

[36] B. Wünsche and R. Lobb. The Visualization of Diffusion Tensor Fields in the Brain. In Int. Conf. on Mathematics and Engineering Techniques in Medicine and Biological Science, METMBS, pages 498-504, 2001.

[37] W. Zhan, E. Stein, and Y. Yang. Mapping the orientation of intravoxel crossing fibers based on the phase information of diffusion circular spectrum. Neuroimage, 23(4):1358-1369, 2004.

[38] S. Zhang, C. Demiralp, and D. Laidlaw. Visualizing Diffusion Tensor MR Images Using Streamtubes and Streamsurfaces. IEEE T Vis Comput Gr, 9(4):454-462, 2003.

[39] L. Zhukov and A. Barr. Oriented Tensor Reconstruction: Tracing Neural Pathways from Diffusion Tensor MRI. In IEEE Visualization, 2002.

[40] M. Zöckler, D. Stalling, and H.-C. Hege. Interactive visualization of 3Dvector fields using illuminated stream lines. In IEEE Visualization, pages 107-113, 1996. 